divided into equal halves; the left weighed 641.5 grammes, the right 684.2 grammes, giving a difference of 42.2 grammes in favour of the right hemisphere. The central ganglia were normal in appearance. The spinal cord was removed from the cervical enlargement to its eatdal termination; it was of firm consistence throughout, and presented no gross lesions. It was divided into segments, and, with portions of the right hemisphere of the brain, the pons and medulla, was hardened in iodised spirit and bichromate of potash. On carefully removing the membranes from the pons and medulla, the right olivary body was found to have lost its plumpness and fulness, the difference between it and its fellow being well marked. As can be seen in this cast of the organ, the furrow dividing it from the anterior pyramid has disappeared, and the position of the body is marked by a few longitudinal striz. On cutting the organ across at the lowest level of the corpora olivaria, the nucleus of the right was seen to be reduced to an oval mass, while that of the left preserved its convolutions, as is depicted in this drawing. This may be simply due to the left nycleus extending farther down than the right, and thus the section would have passed higher in the former than in the latter, and consequently have shown more of the left.

Microscopical Examination.-(a) Pia Mater. The surface of the mem. brane and vessels was covered with densely crowded nuclei ; in some instances so closely packed as to obscure the coats of the latter. Where the coats of the vessels could be distinguished, the muscular layer was apparently unaffected. Scattered also over the surface of the membrane and on the vessels were aggregations of clear disc-like bodies, slightly or not at all tinted by carmine and quite transparent, varying in size from a minute dot to a disc as large again as a red blood-corpuscle, and forming rosettes or irregular groups. These bodies appear to me to be free from globules of fat or oil, and only in one other case have I met with anything like them. (b) Brain. Teased preparations of the fresh tissue revealed the larger pyramidal cells rounded at their basal angles, deprived of the processes springing therefrom, and for the most part loaded with granules. Groups of discs quite similar to those in the pia mater were observed here also, and a compressed section in the fresh condition showed the same appearances. In several specimens taken from the apices of the ascending frontal and parietal convolutions, isolated examples of giant-cells, closely resembling those of the anterior horn of the spinal cord, were visible ; these, however, I could not demonstrate in prepared sections from the same region. In the latter, the arterioles were seen to be varicose, surrounded with connective tissue closely covered with nuclei, and surrounded with particles of yellow hæmatoidin. In transverse sections, the perivascular spaces were somewhat dilated, and contained many nuclei, which formed a bead-like ring around the vessel within. The ganglion-cells had partially lost their regular arrangement, and were in various stages of pigmentary degeneration, and here also the fat-globules were found. The superficial layers of the neuroglia were loose and reticulated; the deeper finer and closer, containing numerous minute nuclei. The capillary vessels were either more numerous or more distinctly seen than is ncrmally the case. (c) Spinal Cord, including the Medulla Oblongata. A transverse section of the medulla, at its junction with the pons, showed the nucleus of the left olivary body to have extended higher than that of the right. Numerous sections through the different parts of this organ from above downwards showed the vessels generally to be thickened and covered with free nuclei, as in the pia mater and brain. The cells of the hypoglossal nuclei bilaterally were diminished in number, many of those remaining being in various stages of degeneration, some being pigmented so as to resemble those of the locus caruleus of the crus cerebri. In one section through the right nucleus, a large vessel was seen containing several thrombi, completely filling its canal and passing into small branches proceeding from it (specimen exhibited). The cells of the nucleus of the vagus and glosso-pharyngeal were relatively less affected than those of the hypoglossal nucleus. The cells of the corpus dentatum of the olivary bodies were throughout slightly pigmented and surrounded in parts by clear spaces. The superficial layer of grey matter on the floor of the fourth ventricle contained masses of nuclei, which, in some sections, had passed deeper. The anterior and external bundles of nerve-fibres did not appear much affected. The central canal, for a short distance below its opening in the fourth ventricle, was patent; lower down, it was obliterated by proliferated nuclei. In the cervical region of the cord, the pia mater, where present in the sections, was, like that on the brain, excessively nucleated, the vessels being similarly affected. The perivascular spaces surround ing those in the neighbourhood of the central canal were widely dilated and eroded at their edges; the latter was, as above, obliterated, and this condition prevailed throughout the entire cord. Scattered in and round the anterior and posterior grey cornua, being especially locatod in the anterior horns, were numerous spots of yellow exudation, appa. rently infiltrating the tissues in which they were placed and blending with the latter. These islets of exudation were most numerous in the grey matter, but also occurred in the white columns. They were en. tirely unaffected by either carmine or aniline blue-black, and were either structureless or finely granular, and could be seen encroaching on and destroying the ganglion-cells (specimens exhibited). These spots somewhat resembled miliary sclerosis, but were of $\cdot a$ more decided yellow colour, not so distinctly separated from the surrounding tissue, and did not possess the lustrous pearly appearance seen in the latter. In the lateral columns in the dorsal region, they were very numerous, being situated externally to the posterior cornua in their vicinity: Several vessels were also observed apparently affected with this exudation, which surrounded and partially obscured them; giving rise to a gap in the equable colouration by carmine (specimen exhibited). The ganglion-cells of the anterior cornua were selectively affected with pig. mentary degeneration and atrophy, those in the upper and middle dorsal, lower lumbar, and sacral regions being most extensively affected. None of the affected cells were reduced to masses of brown granules, being rather rounded, destitute of processes, surrounded entirely by clear circular spaces, clouded, and often finely granular in their interior, with yellow edges. In several instances, the body of the cell appeared to have quite degenerated, the outline remaining from which the origin of the processes could be traced (specimen exhibited). The posterior cornua were deeply coloured, especially the substantia gela. tinosa, the nerve-roots beyond this being much less so. Numerous minute nuclei were scattered through them. In some parts, the peri. vascular canals were so dilated, as to almost separate the anterior from the posterior cornua. The white columns were affected almost equally throughout the entire cord, and generally in the different segments of the section. In the peripheral layers, the axis-cylinders of the nervefibres were crowded together, the circular outlines of the medullary sheaths having in great part disappeared. The connective tissue was closer, and more finely molecular than in the former cases, and dotted with nuclei. In the deeper layers, the normal appearance was more preserved, the anterior columns, at each side of the anterior fissure (columns of Tiirk), being, however, exceptions to this, the deep por tions in front of the grey columns being coarse and reticulated (specimen exhibited). In none of the sections of the spinal cord were there seen any of the groups of globules noticed in the pia mater and brain. Transverse sections of some of the nerves running in the sheath of pia mater showed the axis-cylinders of the fibres to have entirely disappeared; the circular outlines presented normally by the cut ends of the different nerve-strands were irregular in outline and size, some being large, others very small and crowded together; the supporting structure was coarse, in some parts more densely coloured and fibroid than in others; while the investing sheath was thickened and nucleated.

Summary. - Fibrosis of vessels, with nuclear proliferation on and in their coats; overgrowth and granular condition of the connective tissue ; atrophy of nerve-fibres; atrophy and degeneration of ganglion-cells of nuclei of hypoglossal and, to a less extent, of the vagi and glosso. pharyngeal in the medulla and of the anterior cornua in the spinal cord, those in the dorsal region being most affected ; obliteration of the central canal, and marked dilatation throughout of the perivascular spaces ; atrophy of the spinal nerve-fibres.

$$
\text { [To be concluded.] }
$$

\section{THE THEORY OF DISINFECTION.}

BY E. BUCHANAN BAXTER, M.D.,

Professor of Materia Medica and Therapeutics in King's College, etc.

IN a paper on "The Chemical Theory of Contagium compared with the Corpuscular Theory", which appears in last week's Journal, Dr. Notter makes some comments on my "Report on an Experimental Study of Certain Disinfectants", which was published in 1875 . It is not my intention to enter into any controversy on the very interesting subject to which Dr. Notter's paper is devoted; but, as he appears to have altogether misapprehended my meaning in some rather important particulars, and as his paper will doubtless be read by many who have never heard of mine, I trust that room will be afforded me for an explanation.

In the first place, I did not, as Dr. Notter seems to imply, advance the statement that the "infective principle is neither soluble nor diffusible" on my own authority. It is a legitimate, nay, an unavoid. able, deduction from the well-known experiments of Professor Chauveau and Dr. Burdon Sanderson on the contagia of small-pox, cow-pox, glanders, and ovine variola. It is, of course, open to any one to urge 
that the evidence thus furnished is inadequate - that we are not justified in extending to all contagia what has been proved to be true of some only; but, until evidence of the same order and of equal weight has been brought forward on the other side-and I am not aware that this has yet been done-we are surely entitled, at any rate provisionally, to accept the conclusion in question.

Again, the statement that the infective principle is probably nonvolatile, is not, as the reader of Dr. Notter's remarks might fairly suppose, a mere hypothesis or inference. It rests on experimental evidence obtained in the case of small-pox, ovine variola, and cattleplague. (A brief account of the experiments is given on page 219 of my Report.) In short, the assertion that the contagia of certain communicable diseases are neither soluble, diffusible, nor volatile, is grounded on a substantial basis of observed facts; it is not what Magendie was so fond of stigmatising as a "vuc de resprit".

All this, however, only goes to show that contagium is particulate; it does not show that it consists of living "germs". It is quite com. patible with the belief that the virus of infective inflammation is "simply fibrinous exudation, which, after coagulation, has acquired poisonous properties". Whatever may have been my opinion on the subject of "germs", I was careful to keep it well apart from my investigation.

This brings me to the very point to which I wish to draw attention. So far from making the above-mentioned facts-which Dr. Notter terms my "questionable premisses"- -the starting-point of my inquiry, I took great pains to show that the method of research which I ventured to advocate for the determination of the relative value of disinfectant agents differed from those previously followed, in yielding results wholly independent of any theory concerning the intimate nature of the contagia. In this complete independence I conceived its special value to consist. Conclusions arrived at in accordance with the principles laid down in my Report, would lose none of their force were the "chemical" theory, which Dr. Notter is inclined to favour, universally accepted as the true one.

The essential nature of the itfective principle is a problem of vast importance and corresponding difficulty. Were it necessary to solve it before trying to ascertain the value of our disinfectant measures, our hands might remain tied for a long time. I did my best to show that the obstacle might be turned, as it could not be surmounted.

\section{ON CASES OF PLEURITIC EFFUSION.*}

Bg E. MARKHAM SKERRITT, B.A., M.D.Lond., M.R.C.P., Fellow of University College, London; Lecturer on Medicine and on Pathological Anatomy at the Bristol Medical School; Physician to the Bristol General Hospital.

In the present paper, I prupose to bring before you the results of two cases of empyema treated antiseptically, and also to give details of two cases of pleuritic effusion presenting special difficulties or sources of danger.

In considering the treatment of empyema, we have first to answer the question, Is it better to operate, or to let the pus find its own way out of the chest? I do not discuss the possible cure by absorption, as that is at best but a rare occurrence, and one on which we cannot calculate. In what directions may the pus be discharged spontaneously? Most often, either externally or through the lung by bronchial fistula ; sometimes, in other most undesirable directions. Suppose the discharge to be by external fistula ; the pus, before it escapes, burrow's amongst the superficial tissues, often surrounding one or more of the ribs at the site of pointing; hence there often results necrosis of the rib, part of which lies bare in the midst of the pus. Thus there is added chronic bone-disease in a patient who is probably specially prone to the ill effects of such a condition. Again, a spontaneous opening is frequently in the wrong place; instead of being low down so that the cavity can drain, it is commonly high up, in the front of the chest, in the third or fourth space. Next, discharge through the lung is certainly undesirable, for the pus has then to be all pumped up if I may use the expression) by cough, and must pass through a large tract of lung-tissue -a process at once injurious to the lung and most wearying to the patient. The rapid relief and recovery that may follow an artificial external opening in such a case are surprising.

If, then, we may conclude that a spontaneous opening is not a good thing, and that absorption of the pus is not to be expected, it follows that we must remove the fluid by operation. We may do this by means of : 1. Simple tapping or aspiration; 2. Establishing an external fistula.
In most cases, simple aspiration or tapping will not effect a cure, or, if it do, will not until it has been repeated again and again. It is true that, in exceptional cases-and especially in children-one or two tappings may cure the patient; but, generally, the history will be a long series of tappings and fillings of the chest, while the patient is losing ground and the lung is becoming more or less permanently disabled. Therefore, in the majority of cases of empyema, we do well to follow the principle adopted by Nature; that is, to let out the pus by a permanent opening. Why do we hesitate to do this? For the same reason that we used to hesitate to open a psoas abscess; knowing that while the abscess was closed, putrefaction would not occur, but that the opening of the cavity would be the signal for its contents to decompose, and for hectic to set in. An empyema is a large abscess, with an enormous extent of wall ; and the fear of making a free onening was due to the dangers of admitting air into the pleural cavity and setting up putrefaction-a state of things only to be remedied by regularly washing out the cavity; this latter proceeding being both very troublesome and exhausting to the patient and also not devoid of immediate risk to life. I believe that we have no longer cause to fear a frec opening, but that we may make one with perfect confidence, relying upon Lister's antiseptic method to prevent those ill results that were formerly dreaded.

In a paper read before this Branch of the Association, I have previously advocated the antiseptic treatment of empyema (BRITISH MEDiCAL JOURNAL, 1876, vol. ii, p. 109), and have argued that thus we secure all the advantages of a free opening, without any of its disadvantages.

It has been objected that cases of empycma treated antiseptically are as yct too few to admit of generalisation from them; but to this I reply, that the generalisations have been made in the region of surgery in general, and that we merely apply to the case of an empyema the gencral principle that the antiseptic treatment will prevent putrefaction in an open wound. My early surgical experience made me a firm believer in Lister's system, and I only apply to the case of an empyema exactly those principles that would guide me in the treatment of a psoas abscess. What we want in either case is to empty the cavity so that the walls can come together, and at the same time to ward off decomposition. We attain our end by adopting Lister's method.

I will now give a brief outline of two cases of empyema treatment an!iseptically.

The first patient was a deaf and dumb boy, aged I 7 , admitted into the Bristol General Hospital on October Ioth, 1875, said to have been ill one week, although it was evident that the effusion was of much longer standing. There were the usual signs of left pleuritic effusion. Symptoms of hectic became marked, the evening temperature ranging from Ior deg. to $103 \mathrm{deg}$, , or higher, and the morning temperature going down to normal, or helow. Accordingly, on November roth, the chest was aspirated and sixteen ounces of sweet pus were drawn off. After this, the hectic symptoms gradually disappeared, the temperature becoming normal by December 8th (about one month later), and then remaining subnormal. At the same time, however, the physical signs did not materially improve; and if I had depended upon them, I should have said that the chest still contained pus, but I relied upon the temperature-a temperature absolutely and permanently normal or subnormal -as a sure indication that no fresh pus had formed. As the event proved, I was wrong; but I believe it is a most unusual thing for the temperature to follow the course it did here. It is not very uncommon for aspiration to set up hectic when pus previously existed without; but when hectic has once accompanied empyema and the pus has been removed by aspiration, I am under the impression that it is an unprecedented thing for the temperature to remain normal when the pus collects again. The patient was in the hospital for two months longer, during which time his general health improved and the temperature continued subnormal. After being out for about a fortnight, he was readmitted on March 29th, 1876 ; there was then much expectoration of pus, and the temperature was normal.

On April 6th, ten ounces of offensive pus were drawn off by aspiration; but as the excessive expectoration, pointing to bronchial fistula, continued, ten days later a tube was inserted in the ninth interspace, in a line with the angle of the scapula, and antiseptic dressings were applied. The discharge rapidly diminished, and in a week the sputa had become scanty, thin, and watery, instead of purulent. At the same time, the gencral health improved rapidly. On one occasion, the tube slipped out and pus collected in the chest; this was at once shown by gencral discomfort, and by a return of the purulent sputa; but as soon as the channel was restored, these unfavourable symptoms disappeared. A little discharge continued for a long time, but the cavity ultimately closed in October. The boy had now become quite fat; on admission, he weighed sixty-nine pounds, and when sent out 